\title{
Unsteady Hydromagnetic Rotating Flow through an Oscillating Porous Plate Embedded in a Porous Medium
}

\author{
I. Khan, ${ }^{1,2}$ A. Khan, ${ }^{1}$ A. Farhad, ${ }^{1}$ M. Qasim, ${ }^{3}$ and S. Sharidan ${ }^{1}$ \\ ${ }^{1}$ Department of Mathematical Sciences, Faculty of Science, Universiti Teknologi Malaysia, 81310 Skudai, Malaysia \\ ${ }^{2}$ College of Engineering, Majmaah University, P.O. Box 66, Majmaah 11952, Saudi Arabia \\ ${ }^{3}$ Department of Mathematics, COMSATS Institute of Information Technology, Park Road, Chak Shahzad, Islamabad 44000, Pakistan
}

Correspondence should be addressed to S. Sharidan; sharidan@utm.my

Received 23 July 2013; Revised 2 October 2013; Accepted 9 October 2013

Academic Editor: Tirivanhu Chinyoka

Copyright (C) 2013 I. Khan et al. This is an open access article distributed under the Creative Commons Attribution License, which permits unrestricted use, distribution, and reproduction in any medium, provided the original work is properly cited.

\begin{abstract}
This paper investigates unsteady hydromagnetic flow of a viscous fluid in a rotating frame. The fluid is bounded by an oscillating porous plate embedded in a porous medium. The Laplace transform and Fourier sine transform methods are employed to find the exact solutions. They satisfy all imposed initial and boundary conditions and as special cases are reduced to some published results from the literature. The graphical results are plotted for different values of pertinent parameters and some interesting conclusions are made.
\end{abstract}

\section{Introduction}

Stokes' problem for the flow of an incompressible, viscous fluid over an oscillating plane serves as a benchmark in the literature of fluid dynamics [1]. It admits an exact analytical solution. The Stokes' problem not only is of fundamental theoretical interest but also occurs in many applied problems [2]. The transient solutions in terms of tabulated functions for the flow of viscous fluid due to oscillating boundary have been expressed by Panton [3]. Later, in 2000, Erdogan [4] studied the unsteady flow of Newtonian fluid past an oscillating horizontal plane wall and obtained the exact solutions. Fetecau et al. [5] presented new exact solutions corresponding to Stokes' second problem for both small and large times. Erdoğan and Imrak [6] made comparative study for the solution of Stokes' second problem by employing two different transform methods. They expressed transient solutions in terms of tabulated functions.

On the other hand, flow through porous media is very prevalent in nature and, therefore, has become a principal interest of researchers in many scientific and engineering studies. For example, one can refer to the books of Pop and Ingham [7], Ingham et al. [8], Ingham and Pop [9], Vafai [10], and Nield and Bejan [11] for the detailed literature on this topic. Moreover, the rotating flow through porous media has been the subject of many studies in the last few decades due to their wide range of applications in cosmological and geophysical fluid dynamics, astrophysics, meteorology, petroleum, and hydrology to study the movement of underground water (Jana et al. [12]). According to Hayat et al. [13], the analysis of the effects of rotation and magnetic field in fluid flows has been an active area of research because of its geophysical and technological applications such as the MHD power generator and boundary layer flow control. Based on this motivation, Das et al. studied in [14] the simultaneous effects of rotation and magnetic field on the flow of a second grade fluid between two parallel plates. Bearing in mind the importance of MHD and porosity in a rotating fluid, Hayat et al. $[15,16]$, Abelman et al. [17], Sahoo et al. [18], Seth et al. [19], and Farhad et al. [20] studied the hydromagnetic flow of rotating fluids in porous media. Recently, Jana et al. [21] studied the unsteady flow of viscous fluid through a porous medium bounded by a porous plate in a rotating system.

In order to further discuss the work of Jana et al. [21] and to make closer relations between this study and practical engineering, we study in this work hydromagnetic rotating flow of a viscous fluid bounded by an oscillating porous plate embedded in a porous medium. The governing mathematical problem is solved by using the Laplace transform and Fourier sine transform methods. The expressions for velocity and skin 
friction in case of cosine and sine oscillations of the plate are obtained. The analytical results are plotted and discussed. The results of Jana et al. [21] are recovered as a special case from our obtained solutions. The present study is useful in astronomy to study the rotating motion of astrophysical objects such as magnetic stars.

\section{Mathematical Formulation of the Problem}

Consider the unsteady flow of an incompressible viscous fluid occupying the upper porous half-space of the $(x, y)$ plane. The fluid is bounded by a porous plate at $z=0$ such that the positive $z$-axis is taken normal to the plate and the $x$ axis is taken parallel to the plate. We consider the hydromagnetic flow induced in the fluid in the presence of a uniform magnetic field of strength $B_{0}$ applied in a direction normal to the plate by means of the plate oscillations in its plane (Hayat et al. [13]). The electric field due to polarization of charges is neglected. Both of the fluid and plate are in a state of rigid body rotation with constant angular velocity $\boldsymbol{\Omega}=\Omega \widehat{\mathbf{k}} ; \widehat{\mathbf{k}}$ is a unit vector in the $z$-direction. The geometry of the problem is shown in Figure 1. Initially, for $t \leq 0$, both of the fluid and plate are at rest. At time $t=0^{+}$, the lower plate suddenly starts to move in its own plane with oscillating velocity in the flow direction. Under these assumptions, the equations of momentum in a rotating frame along the $x$ - and $y$-directions are [18-20]

$$
\begin{array}{r}
\frac{\partial u}{\partial t}-w_{0} \frac{\partial u}{\partial z}-2 \Omega v=v \frac{\partial^{2} u}{\partial z^{2}}-\frac{\nu \phi}{k} u-\frac{\sigma B_{0}^{2}}{\rho} u \\
z, t>0, \\
\frac{\partial v}{\partial t}-w_{0} \frac{\partial v}{\partial z}+2 \Omega u=v \frac{\partial^{2} v}{\partial z^{2}}-\frac{\nu \phi}{k} v-\frac{\sigma B_{0}^{2}}{\rho} v \\
z, t>0
\end{array}
$$

where $u$ and $v$ are the velocity components in $x$ - and $y$ directions, $w_{0}$ is the suction velocity at the plate, $v$ is the kinematic viscosity, $t$ is the time, $\sigma$ is the electrical conductivity, $B_{0}$ is the applied magnetic field, $\rho$ is the fluid density, $\phi$ is the porosity, and $k$ is the permeability of the porous medium.

The corresponding initial and boundary conditions are

$$
\begin{gathered}
u=v=0 ; \quad t \leq 0, \\
u=U H(t) \cos \left(\omega_{0} t\right) \quad \text { or } U \sin \left(\omega_{0} t\right) ; \\
v=0 ; \quad \text { at } z=0 ; t>0, \\
u \longrightarrow 0 ; \quad v \longrightarrow 0 \text { as } z \longrightarrow \infty ; t>0,
\end{gathered}
$$

in which $U$ is the constant plate velocity, $\omega_{0}$ is the frequency of oscillation of the plate, and $H(t)$ is the Heaviside function.

Defining $F=u+i v$, the above system of equations reduces to

$$
\begin{array}{r}
\frac{\partial F}{\partial t}-w_{0} \frac{\partial F}{\partial z}+2 i \Omega F=\nu \frac{\partial^{2} F}{\partial z^{2}}-\frac{\nu \phi}{k} F-\frac{\sigma B_{0}^{2}}{\rho} F \\
z, t>0
\end{array}
$$

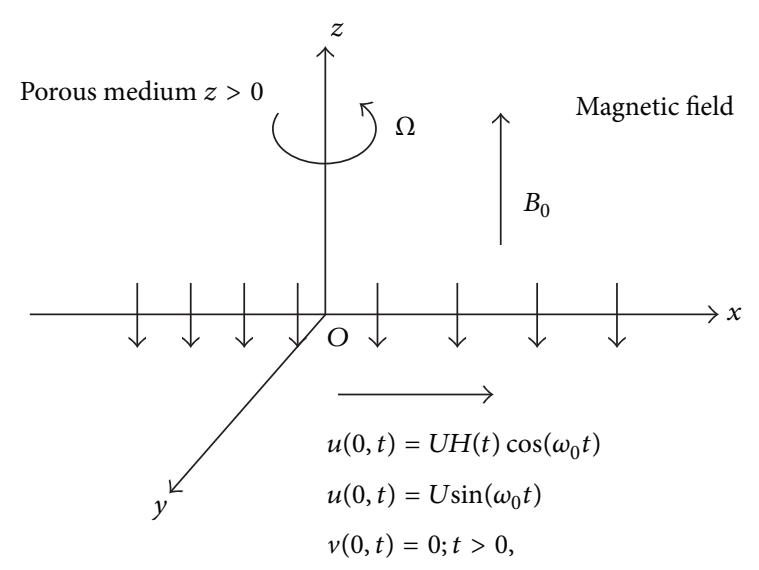

FIgURE 1: Geometry of the problem.

$$
\begin{gathered}
F(z, 0)=0 ; \quad t \leq 0, \\
F(0, t)=U H(t) \cos \left(\omega_{0} t\right) \quad \text { or } U \sin \left(\omega_{0} t\right) ; \quad t>0 \\
F \longrightarrow 0 \quad \text { as } z \longrightarrow \infty ; t>0 .
\end{gathered}
$$

Introducing the following dimensionless variables (see Jana et al. [21]):

$$
G=\frac{F}{U}, \quad \xi=\frac{z U}{v}, \quad \tau=\frac{U^{2} t}{v}, \quad \omega=\frac{\omega_{0} v}{U^{2}},
$$

the dimensionless problem takes the following form:

$$
\begin{gathered}
\frac{\partial G}{\partial \tau}-S \frac{\partial G}{\partial \xi}+2 i \eta G=\frac{\partial^{2} G}{\partial \xi^{2}}-M^{2} G-\frac{1}{K} G \\
G(\xi, 0)=0 ; \quad \tau \leq 0, \\
G(0, \tau)=H(\tau) \cos (\omega \tau) \quad \text { or } \quad \sin (\omega \tau) ; \quad \tau>0, \\
G(\infty, \tau)=0 ; \quad \tau>0, \\
M^{2}=\frac{\sigma B_{0}^{2} \nu}{\rho U^{2}}, \quad \frac{1}{K}=\frac{\nu^{2} \phi}{k U^{2}}, \quad \eta=\frac{\Omega \nu}{U^{2}}, \quad S=\frac{w_{0}}{U},
\end{gathered}
$$

where $M, K, \eta$, and $S$ are the magnetic, permeability, rotation, and suction parameters, respectively.

\section{Solution of the Problem by Using Laplace Transform}

Taking the Laplace transform of (7) and using the initial condition (8), we get the following transformed differential equation:

$$
\begin{aligned}
& \frac{d^{2} \bar{G}(\xi, q)}{d \xi^{2}}+S \frac{d \bar{G}(\xi, q)}{d \xi} \\
& \quad-\left[q+M^{2}+\frac{1}{K}+2 i \eta\right] \bar{G}(\xi, q)=0,
\end{aligned}
$$


where $q$ is the transform parameter. In view of the boundary conditions (9) and (10), the Laplace transform $\bar{G}(\xi, q)$ of $G(\xi, \tau)$ has to satisfy the following conditions:

$$
\begin{gathered}
\bar{G}(0, q)=\frac{q}{q^{2}+\omega^{2}} \quad \text { or } \quad \bar{G}(0, q)=\frac{\omega}{q^{2}+\omega^{2}} \\
\bar{G}(0, q)=0 .
\end{gathered}
$$

Solution of (12) under the boundary conditions (13) and (14) yields

$$
\bar{G}_{c}(\xi, q)=\frac{1}{2} e^{-\xi S / 2}\left\{\frac{1}{q+i \omega} e^{-\xi \sqrt{q+a}}+\frac{1}{q-i \omega} e^{-\xi \sqrt{q+a}}\right\},
$$

respectively,

$$
\bar{G}_{s}(\xi, q)=\frac{1}{2 i} e^{-\xi S / 2}\left\{-\frac{1}{q+i \omega} e^{-\xi \sqrt{q+a}}+\frac{1}{q-i \omega} e^{-\xi \sqrt{q+a}}\right\},
$$

where the subscripts $c$ and $s$ denote the cosine and sine oscillations of the plate and

$$
a=\frac{S^{2}}{4}+\frac{1}{K}+M^{2}+2 i \eta
$$

Now, in order to find the inverse Laplace transform of (15) and (16), we use formula (A4) from the Appendix of Farhad et al. [20]; hence, we get the following solutions:

$$
\begin{aligned}
& G_{c}(\xi, \tau) \\
& =\frac{H(\tau) e^{-\xi S / 2}}{4} \\
& \quad \times\left[e ^ { - i \omega \tau } \left\{e^{-\xi \sqrt{a-i \omega}} \operatorname{erf}\left(\frac{\xi}{2 \sqrt{\tau}}-\sqrt{(a-i \omega) \tau}\right)\right.\right. \\
& \left.\left.\quad+e^{\xi \sqrt{a-i \omega}} \operatorname{erf}\left(\frac{\xi}{2 \sqrt{\tau}}+\sqrt{(a-i \omega) \tau}\right)\right\}\right] \\
& \quad+\frac{H(\tau) e^{-\xi S / 2}}{4} \\
& \quad \times\left[e ^ { i \omega \tau } \left\{e^{-\xi \sqrt{a+i \omega}} \operatorname{erf} c\left(\frac{\xi}{2 \sqrt{\tau}}-\sqrt{(a+i \omega) \tau}\right)\right.\right. \\
& \left.\left.\quad+e^{-\xi \sqrt{a+i \omega}} \operatorname{erf} c\left(\frac{\xi}{2 \sqrt{\tau}}+\sqrt{(a+i \omega) \tau}\right)\right\}\right],
\end{aligned}
$$

respectively,

$$
\begin{aligned}
& G_{s}(\xi, \tau) \\
& =\frac{e^{-\xi S / 2}}{4 i} \\
& \quad \times\left[-e^{-i \omega \tau}\left\{e^{-\xi \sqrt{a-i \omega}} \operatorname{erf} c\left(\frac{\xi}{2 \sqrt{\tau}}-\sqrt{(a-i \omega) \tau}\right)\right.\right. \\
& \left.\left.+e^{\xi \sqrt{a-i \omega}} \operatorname{erf} c\left(\frac{\xi}{2 \sqrt{\tau}}+\sqrt{(a-i \omega) \tau}\right)\right\}\right] \\
& +\frac{e^{-\xi S / 2}}{4 i}\left[e ^ { i \omega \tau } \left\{e^{-\xi \sqrt{a+i \omega}} \operatorname{erf} c\left(\frac{\xi}{2 \sqrt{\tau}}-\sqrt{(a+i \omega) \tau}\right)\right.\right. \\
& \left.\left.\quad+e^{\xi \sqrt{a+i \omega}} \operatorname{erf} c\left(\frac{\xi}{2 \sqrt{\tau}}+\sqrt{(a+i \omega) \tau}\right)\right\}\right] .
\end{aligned}
$$

\section{Solution of the Problem by Using Fourier Sine Transform}

Now, applying Fourier sine transform to (12) and using boundary conditions (13) and (14), we obtain

$$
\widetilde{G}_{c}(\zeta, q)=\frac{\sqrt{2}(\zeta+\zeta S) q}{\left(\zeta^{2}+\zeta^{2} S+q+a\right)\left(q^{2}+\omega^{2}\right)},
$$

respectively,

$$
\widetilde{G}_{s}(\zeta, q)=\frac{\sqrt{2}(\zeta+\zeta S) \omega}{\left(\zeta^{2}+\zeta^{2} S+q+a\right)\left(q^{2}+\omega^{2}\right)},
$$

where $\widetilde{G}(\zeta, q)$ denotes Fourier sine transform of $\bar{G}(\xi, q)$.

Now, we take the inverse Fourier sine transform of (20), which yields

$$
G_{c}(\xi, q)=\frac{2 \sqrt{2} q}{\pi\left(q^{2}+\omega^{2}\right)} \int_{0}^{\infty} \frac{(\zeta+\zeta S)}{\left(\zeta^{2}+\zeta^{2} S+q+a\right)} \sin (\zeta \xi) d \zeta .
$$

Taking the inverse Laplace transform of (22), we obtain

$$
\begin{aligned}
G_{c}(\xi, \tau)=\frac{2}{\pi} \int_{0}^{\infty} \frac{\zeta\left(\zeta^{2}+\zeta^{2} S+a\right) e^{-\left(\zeta^{2}+\zeta^{2} S+a\right) \tau}}{\left(\left(\zeta^{2}+\zeta^{2} S+a\right)^{2}+\omega^{2}\right)} \\
\quad \times \sin (\zeta \xi) d \zeta \\
-\frac{2}{\pi} \int_{0}^{\infty} \frac{\zeta S\left(\zeta^{2}+\zeta^{2} S+a\right) e^{-\left(\zeta^{2}+\zeta^{2} S+a\right) \tau}}{\left(\left(\zeta^{2}+\zeta^{2} S+a\right)^{2}+\omega^{2}\right)} \\
\\
\quad \times \sin (\zeta \xi) d \zeta
\end{aligned}
$$




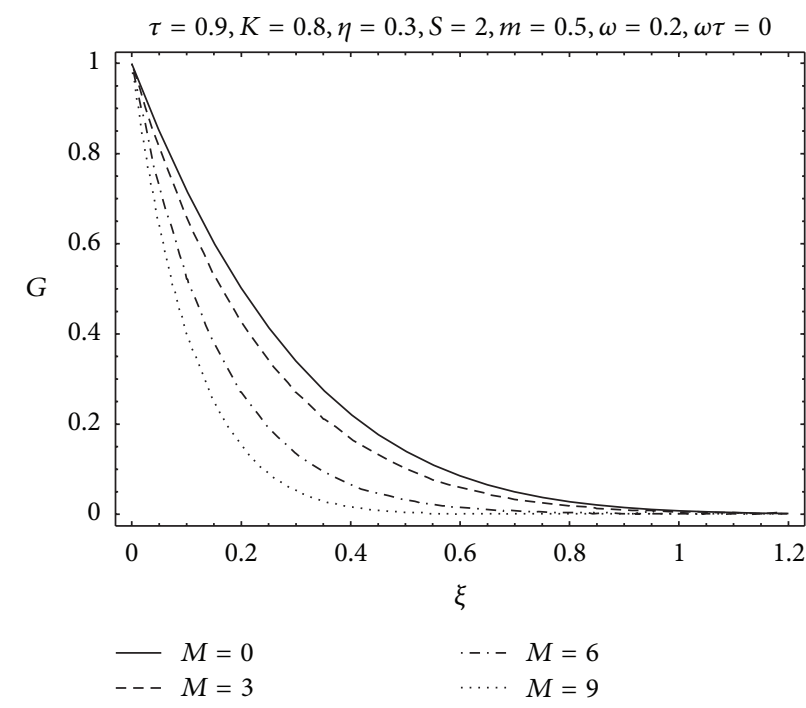

(a)

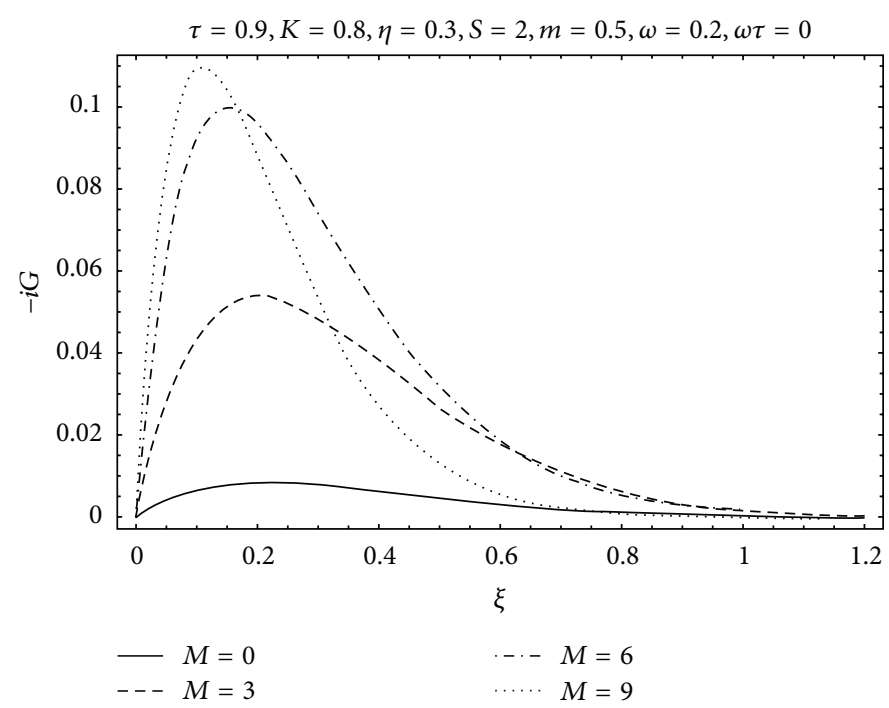

(b)

FIGURE 2: Profiles of velocity for different values of $M$.

$$
\begin{aligned}
& +\frac{2}{\pi} \int_{0}^{\infty} \frac{\zeta\left(\zeta^{2}+\zeta^{2} S+a\right)}{\left(\left(\zeta^{2}+\zeta^{2} S+a\right)^{2}+\omega^{2}\right)} \\
& \times \cos (\omega \tau) \sin (\zeta \xi) d \zeta \\
& +\frac{2}{\pi} \int_{0}^{\infty} \frac{\zeta \omega}{\left(\left(\zeta^{2}+\zeta^{2} S+a\right)^{2}+\omega^{2}\right)} \\
& \times \frac{\sin (\omega \tau) \sin (\zeta \xi) d \zeta}{\pi} \int_{0}^{\infty} \frac{\zeta S\left(\zeta^{2}+\zeta^{2} S+a\right)}{\left(\left(\zeta^{2}+\zeta^{2} S+a\right)^{2}+\omega^{2}\right)} \\
& \times \cos (\omega \tau) \sin (\zeta \xi) d \zeta \\
& +\frac{2}{\pi} \int_{0}^{\infty} \frac{\zeta S \omega}{\left(\left(\zeta^{2}+\zeta^{2} S+a\right)^{2}+\omega^{2}\right)} \\
& \times \sin (\omega \tau) \sin (\zeta \xi) d \zeta .
\end{aligned}
$$

Similarly for the sine oscillation of the boundary we get from (21) the following:

$$
\begin{aligned}
G_{s}(\xi, \tau)= & \frac{2}{\pi} \int_{0}^{\infty} \frac{\zeta \omega e^{-\left(\zeta^{2}+\zeta^{2} S+a\right) \tau}}{\left(\left(\zeta^{2}+\zeta^{2} S+a\right)^{2}+\omega^{2}\right)} \sin (\zeta \xi) d \zeta \\
& -\frac{2}{\pi} \int_{0}^{\infty} \frac{\zeta \omega}{\left(\left(\zeta^{2}+\zeta^{2} S+a\right)^{2}+\omega^{2}\right)} \cos (\omega \tau) \sin (\zeta \xi) d \zeta
\end{aligned}
$$

$$
\begin{aligned}
& +\frac{2}{\pi} \int_{0}^{\infty} \frac{\zeta\left(\zeta^{2}+\zeta^{2} S+a\right) \omega}{\left(\left(\zeta^{2}+\zeta^{2} S+a\right)^{2}+\omega^{2}\right)} \sin (\omega \tau) \sin (\zeta \xi) d \zeta \\
& +\frac{2}{\pi} \int_{0}^{\infty} \frac{\zeta S \omega e^{-\left(\zeta^{2}+\zeta^{2} S+a\right) \tau}}{\left(\left(\zeta^{2}+\zeta^{2} S+a\right)^{2}+\omega^{2}\right)} \sin (\zeta \xi) d \zeta \\
& -\frac{2}{\pi} \int_{0}^{\infty} \frac{\zeta S \omega}{\left(\left(\zeta^{2}+\zeta^{2} S+a\right)^{2}+\omega^{2}\right)} \cos (\omega \tau) \sin (\zeta \xi) d \zeta \\
& +\frac{2}{\pi} \int_{0}^{\infty} \frac{\zeta S\left(\zeta^{2}+\zeta^{2} S+a\right)}{\left(\left(\zeta^{2}+\zeta^{2} S+a\right)^{2}+\omega^{2}\right)} \sin (\omega \tau) \sin (\zeta \xi) d \zeta .
\end{aligned}
$$

The nondimensional shear stresses $\tau_{c}$ and $\tau_{s}$ at the plate $\xi=$ 0 , due to cosine and sine oscillations of the plate, are obtained from (18) and (19) as follows:

$$
\begin{aligned}
\tau_{c}=- & \left(\tau_{x}+i \tau_{y}\right) \\
=- & \frac{H(\tau) e^{-a t-i \omega \tau}}{4 \sqrt{\pi \tau}} \\
\times[ & -4 e^{i \omega \tau}-S \sqrt{\pi \tau} e^{a \tau}-S \sqrt{\pi \tau} e^{a \tau+2 i \omega \tau} \\
& -2 e^{a \tau} \sqrt{(a-i \omega) \pi \tau}-2 e^{a \tau+2 i \omega \tau} \sqrt{(a+i \omega) \pi \tau} \\
& +2 e^{a \tau} \sqrt{(a-i \omega) \pi \tau} \operatorname{erf} c(\sqrt{(a-i \omega) \tau}) \\
& \left.\left.+2 \sqrt{(a+i \omega) \pi \tau} e^{a \tau+2 i \omega \tau} \operatorname{erf} c(\sqrt{(a+i \omega) \tau})\right)\right],
\end{aligned}
$$




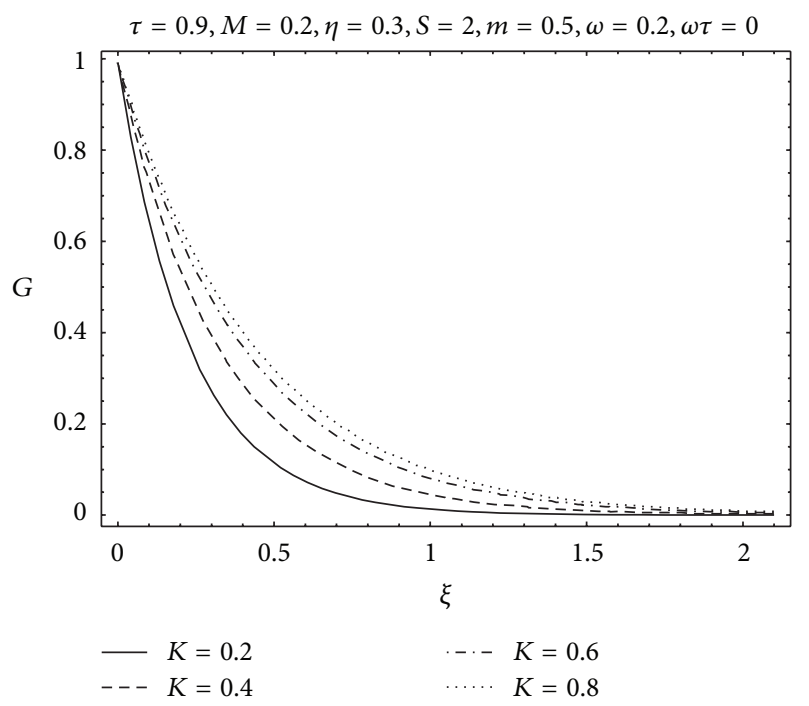

(a)

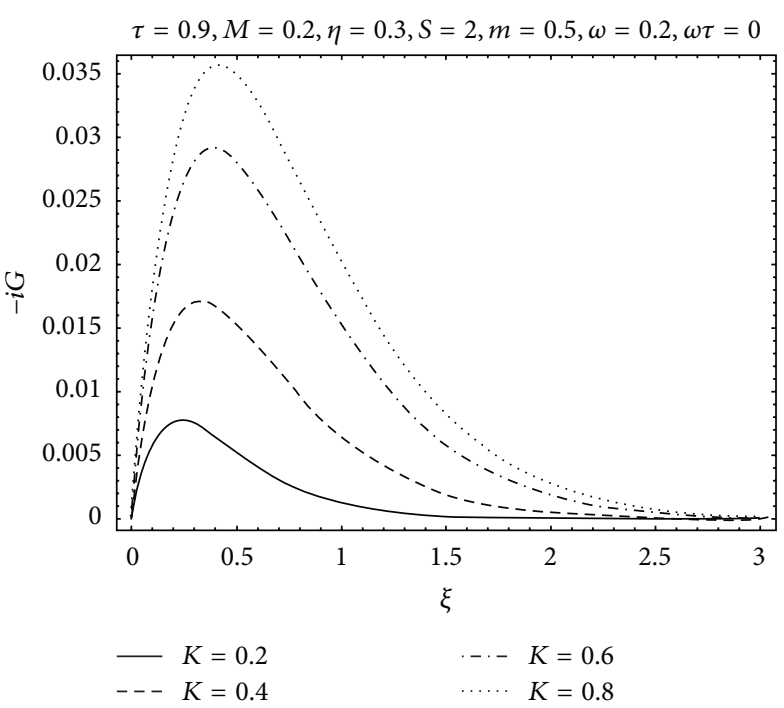

(b)

Figure 3: Profiles of velocity for different values of $K$.

$$
\begin{aligned}
\tau_{s}= & -\left(\tau_{x}+i \tau_{y}\right) \\
= & \frac{i e^{-a t-i \omega \tau}}{4 \sqrt{\pi \tau}} \\
& \times\left[\left(-4 e^{i \omega \tau}-S \sqrt{\pi \tau} e^{a \tau}-S \sqrt{\pi \tau} e^{a \tau+2 i \omega \tau}\right.\right. \\
& -2 e^{a \tau} \sqrt{(a-i \omega) \pi \tau}-2 e^{a \tau+2 i \omega \tau} \sqrt{(a+i \omega) \pi \tau} \\
& +2 e^{a \tau} \sqrt{(a-i \omega) \pi \tau} \operatorname{erf} c(\sqrt{(a-i \omega) \tau}) \\
& \left.\left.+2 \sqrt{(a+i \omega) \pi \tau} e^{a \tau+2 i \omega \tau} \operatorname{erf} c(\sqrt{(a+i \omega) \tau})\right)\right] .
\end{aligned}
$$

\section{Special Cases}

In order to verify the correctness of our obtained solutions, it is important to note that (18) and (19) satisfy the governing equation and the imposed initial and boundary conditions. Further, these solutions are more general comparing to the existing solutions in the literature. In this section, we derive some special cases of these solutions. The solution given by (18) for hydrodynamic fluid $(M=0)$ over an impulsively moved plate $(\omega=0)$ reduces to the following form:

$$
\begin{aligned}
G(\xi, \tau)= & \frac{H(\tau) e^{-\xi S / 2}}{2} \\
& \times\left[\begin{array}{l}
e^{-\xi \sqrt{S^{2} / 4+1 / K+2 i \eta}} \\
\quad \times \operatorname{erf} c\left(\frac{\xi}{2 \sqrt{\tau}}-\sqrt{\left(\frac{S^{2}}{4}+\frac{1}{K}+2 i \eta\right) \tau}\right)
\end{array}\right.
\end{aligned}
$$

$$
\begin{aligned}
& +e^{\xi \sqrt{S^{2} / 4+1 / K+2 i \eta}} \\
& \left.\times \operatorname{erf} c\left(\frac{\xi}{2 \sqrt{\tau}}+\sqrt{\left(\frac{S^{2}}{4}+\frac{1}{K}+2 i \eta\right) \tau}\right)\right],
\end{aligned}
$$

which is identical to the solution given by (13), obtained by Jana et al. [21]. Further, the present solution given by (18) for suddenly moved plate $(\omega=0)$ reduces to the solution (19) obtained by Farhad et al. [20] in the absence of Hall current and slip boundary condition. Hence this provides a useful mathematical check to our calculi.

\section{Results and Discussion}

The exact solutions for the unsteady hydromagnetic flow of viscous fluid bounded by a porous plate in a porous medium are obtained. The analytical results are displayed for various values of emerging parameters such as Hartmann number $M$, permeability parameter $K$, rotation parameter $\eta$, suction parameter $S$, and phase angle $\omega \tau$. Figures 2-6 are plotted for the cosine velocity given by (18). In these figures, (a) and (b) show the real and imaginary parts of velocity.

Figure 2 is prepared to show the variation of velocity for different values of Hartmann number $M$. It is found that the real part of velocity and boundary layer thickness decreases with increasing values of Hartmann number. It is due to the fact that the application of transverse magnetic field results a resistive type force (called Lorentz force) similar to drag force and upon increasing the values of $M$, the drag force increases which leads to the deceleration of the flow. However, it is observed that this behavior is quite opposite for the imaginary part of velocity. Figure 3 reveals that the presence of porous medium increases the resistance to flow thus reducing its 


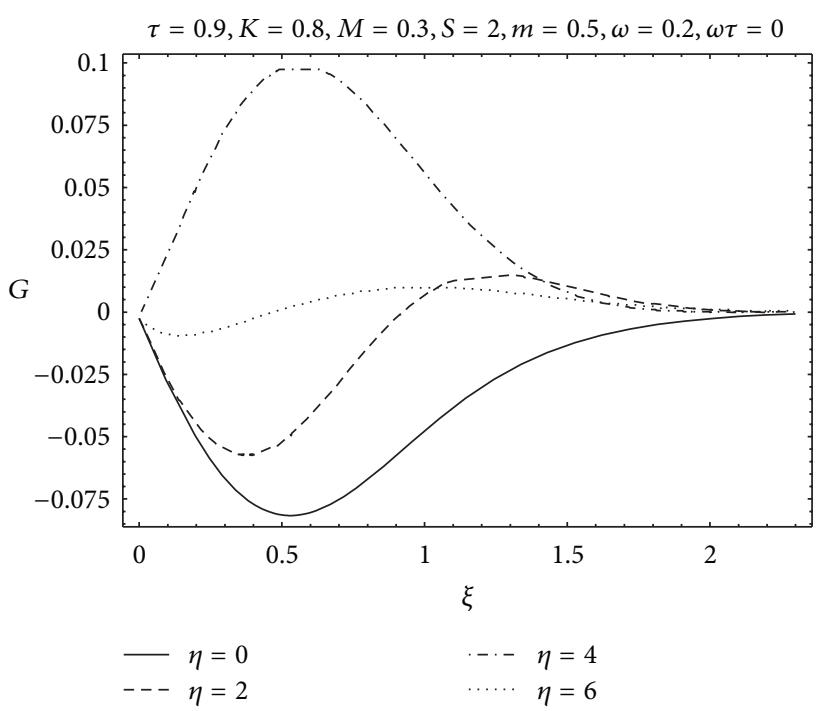

(a)

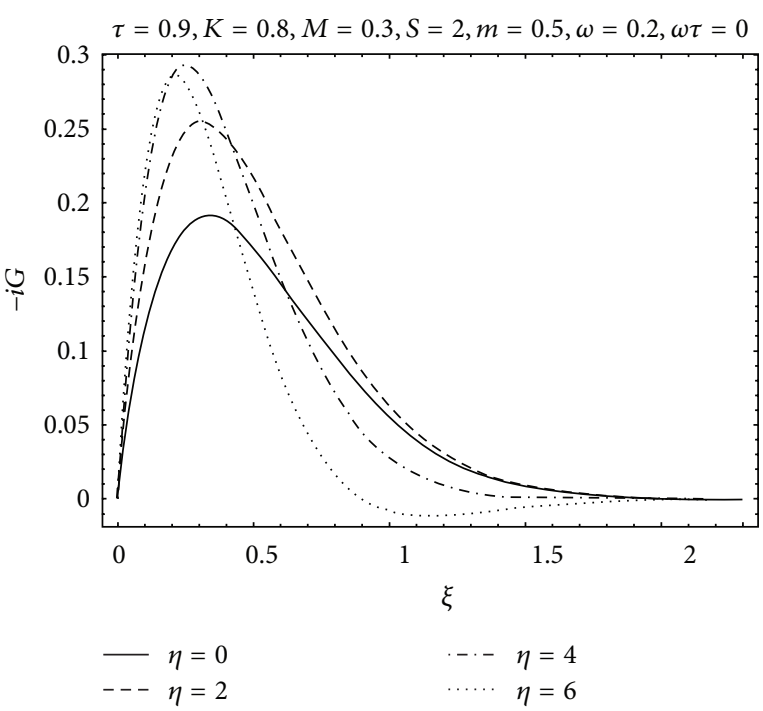

(b)

Figure 4: Profiles of velocity for different values of $\eta$.

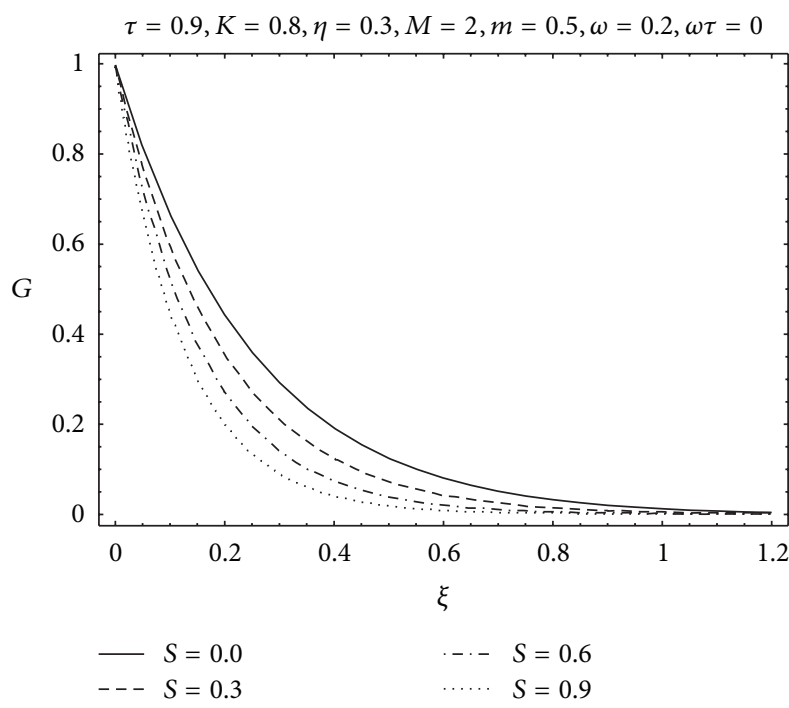

(a)

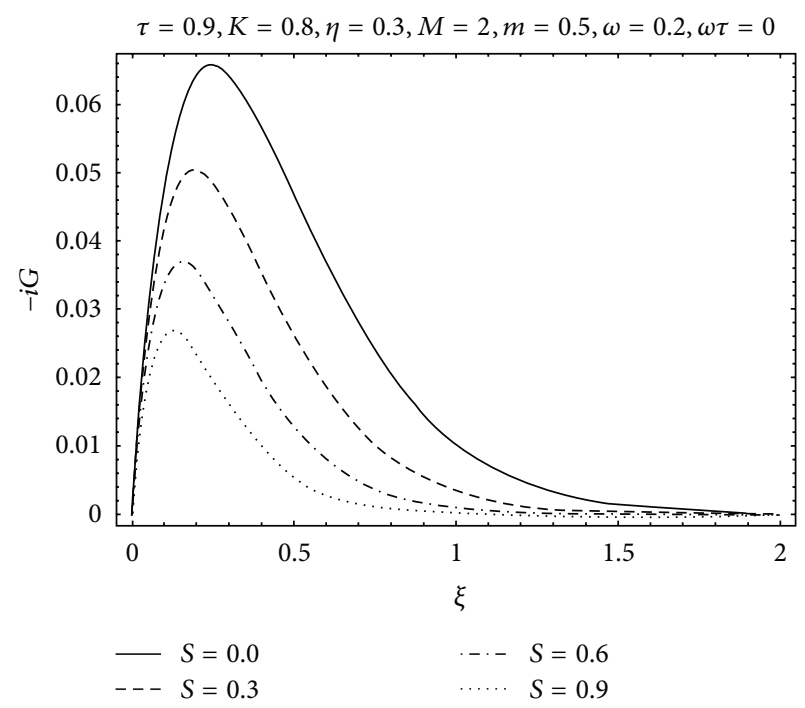

(b)

FIgure 5: Profiles of velocity for different values of $S$.

velocity. It may also be expected due to the fact that increasing values of $K$ reduces the friction forces which assists the fluid considerably to move fast and increases the boundary layer thickness. The graphs for the rotation parameter are plotted in Figure 4. It is observed that the real part of velocity increases when $\eta$ is increased. However, when observed carefully, it is found from Figure 4(b) that the behavior of imaginary part of velocity is more oscillatory in nature. The velocity increases first and then decreases when $\eta$ is increased.

Figure 5 shows the effect of suction parameter $S$ on the flow through a porous medium in a rotating frame. Clearly, the absolute value of velocity and boundary layer thickness for both real and imaginary parts of velocity decrease with an increase in suction parameter. The variation of velocity for different values of phase angle $\omega \tau$ is shown in Figure 6. It is found that the real part of velocity decreases with increasing phase angle $\omega \tau$. Two different values of the phase angle $\omega \tau=$ $0 \pi$ and $\pi / 2$ are chosen. It is interesting to note that, when $\omega \tau=0 \pi$, the real part of velocity is 1 which corresponds to the impulsive motion of the plate. Moreover, for $\omega \tau=\pi / 2$, the real part of velocity is 0 . The absolute value of the imaginary part of velocity is increasing with increasing phase angle $\omega \tau$. Furthermore, for large values of $\xi$, that is, $\xi \rightarrow \infty$, both of the real and imaginary parts of velocity are approaching zero. The graphical behavior in this figure is in accordance with the imposed boundary conditions (3) and (4). Moreover, it 


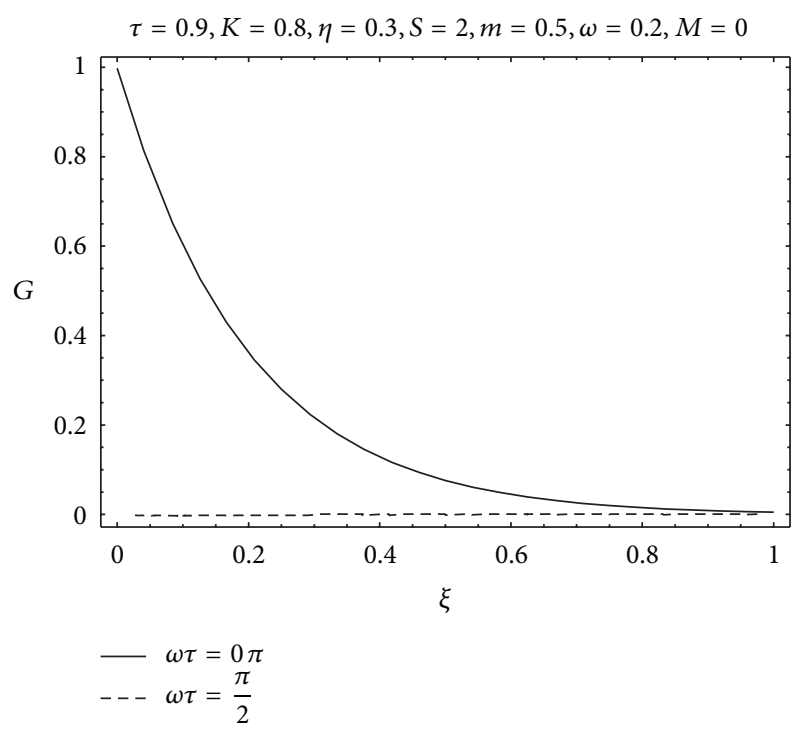

(a)

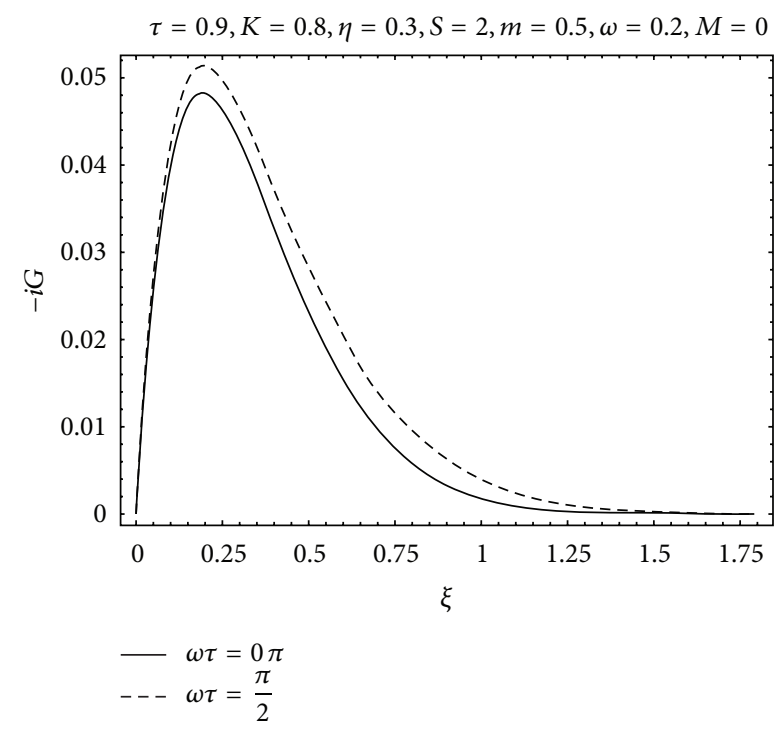

(b)

Figure 6: Profiles of velocity for different values of $\omega \tau$.

is interesting to note from Figure 6 that for a hydrodynamic fluid and impulsive motion of the plate, that is, when $M=0$ and $\omega=0$, the graph for velocity matches with that of Jana et al. [21], which ensures the accuracy of the obtained analytical results.

\section{Conclusions}

The unsteady hydromagnetic rotating flow of viscous fluid bounded by an oscillating porous plate embedded in a porous medium is studied. The Laplace transform and Fourier sine transform methods are used for finding the solutions of the problem. The analytical results for nondimensional velocity and skin friction are obtained. The graphical results are plotted. The results show that velocity increases with increasing rotation parameter and permeability parameter whereas it decreases with increasing Hartmann number, suction parameter, and phase angle. Moreover, as the permeability of the medium increases, the velocity field increases in the boundary layer. Thus we can control the velocity field by introducing porous medium in a rotating system.

\section{References}

[1] H. Schlichting, Grenzchicht Theorie, Braun, Karlsruhe, Germany, 8th ed edition, 1982.

[2] N. Tokuda, "On the impulsive motion of a flat plate in a viscous fluid," Journal of Fluid Mechanics, vol. 33, no. 4, pp. 657-672, 1968.

[3] R. Panton, "The transient solution for Stokes's oscillating plane: a solution in terms of tabulated functions," Journal of Fluid Mechanics, vol. 31, no. 4, pp. 819-825, 1968.

[4] M. E. Erdogan, "Note on an unsteady flow of a viscous fluid due to an oscillating plane wall," International Journal of Non-Linear Mechanics, vol. 35, no. 1, pp. 1-6, 2000.

[5] C. Fetecau, D. Vieru, and C. Fetecau, "A note on the second problem of Stokes for Newtonian fluids," International Journal of Non-Linear Mechanics, vol. 43, no. 5, pp. 451-457, 2008.

[6] M. E. Erdoğan and C. E. Imrak, "On the comparison of the solutions obtained by using two different transform methods for the second problem of Stokes for Newtonian fluids," International Journal of Non-Linear Mechanics, vol. 44, no. 1, pp. 27-30, 2009.

[7] I. Pop and D. B. Ingham, Convective Heat Transfer: Mathematical and Computational Modeling of Viscous Fluids and Porous Media, Pergamon, Oxford, UK, 2001.

[8] D. B. Ingham, A. Bejan, E. Mamut, and I. Pop, Emerging Technologies and Techniques in Porous Media, Kluwer Academic, Dodrecht, The Netherlands, 2004.

[9] D. B. Ingham and I. Pop, Transport Phenomena in Porous Media, Pergamon, Oxford, UK, 2005.

[10] K. Vafai, Handbook of Porous Media, Taylor \& Francis, New York, NY, USA, 2005.

[11] D. A. Nield and A. Bejan, Convection in Porous Media, Springer, New York, NY, USA, 3rd edition, 2006.

[12] M. Jana, S. Das, and R. N. Jana, "Unsteady Couette flow through a porous medium in a rotating system," Open Journal of Fluid Dynamics, vol. 2, pp. 149-158, 2012.

[13] T. Hayat, S. Nadeem, A. M. Siddiqui, and S. Asghar, "An oscillating hydromagnetic non-Newtonian flow in a rotating system," Applied Mathematics Letters, vol. 17, no. 6, pp. 609-614, 2004.

[14] S. Das, S. L. Maji, M. Guria, and R. N. Jana, "Unsteady MHD Couette flow in a rotating system," Mathematical and Computer Modelling, vol. 50, no. 7-8, pp. 1211-1217, 2009.

[15] T. Hayat, C. Fetecau, and M. Sajid, "Analytic solution for MHD Transient rotating flow of a second grade fluid in a porous space," Nonlinear Analysis: Real World Applications, vol. 9, no. 4, pp. 1619-1627, 2008.

[16] T. Hayat, C. Fetecau, and M. Sajid, "On MHD transient flow of a Maxwell fluid in a porous medium and rotating frame," Physics Letters A, vol. 372, no. 10, pp. 1639-1644, 2008.

[17] S. Abelman, E. Momoniat, and T. Hayat, "Steady MHD flow of a third grade fluid in a rotating frame and porous space," Nonlinear Analysis: Real World Applications, vol. 10, no. 6, pp. 3322-3328, 2009. 
[18] S. N. Sahoo, J. P. Panda, and G. C. Dash, "Hydromagnetic oscillatory flow and heat transfer of a viscous liquid past a vertical porous plate in a rotating medium," Indian Journal of Science and Technology, vol. 3, pp. 817-821, 2010.

[19] G. S. Seth, M. S. Ansari, and R. Nandkeolyar, "Unsteady hydromagnetic Couette flow within porous plates in a rotating system," Advances in Applied Mathematics and Mechanics, vol. 2, no. 3, pp. 286-302, 2010.

[20] A. Farhad, M. Norzieha, S. Sharidan, I. Khan, and Samiulhaq, "Hydromagnetic rotating flow in a porous medium with slip condition and Hall current," International Journal of Physical Sciences, vol. 7, no. 10, pp. 1540-1548, 2012.

[21] M. Jana, S. L. Maji, S. Das, and R. N. Jana, "Unsteady flowof viscous fluid through a porous medium bounded bya porous plate in a rotating system," Journal of Porous Media, vol. 13, no. 7, pp. 645-653, 2010. 


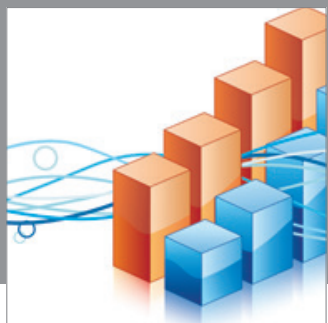

Advances in

Operations Research

mansans

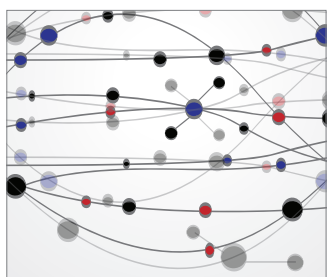

The Scientific World Journal
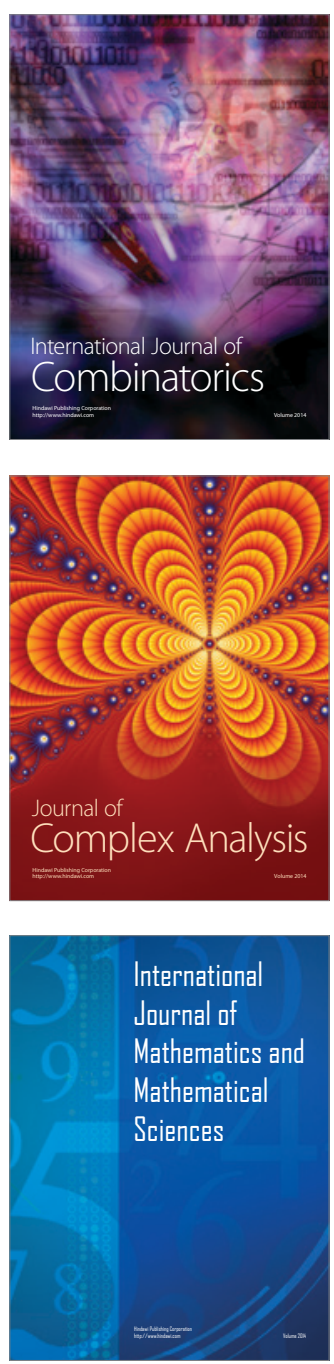
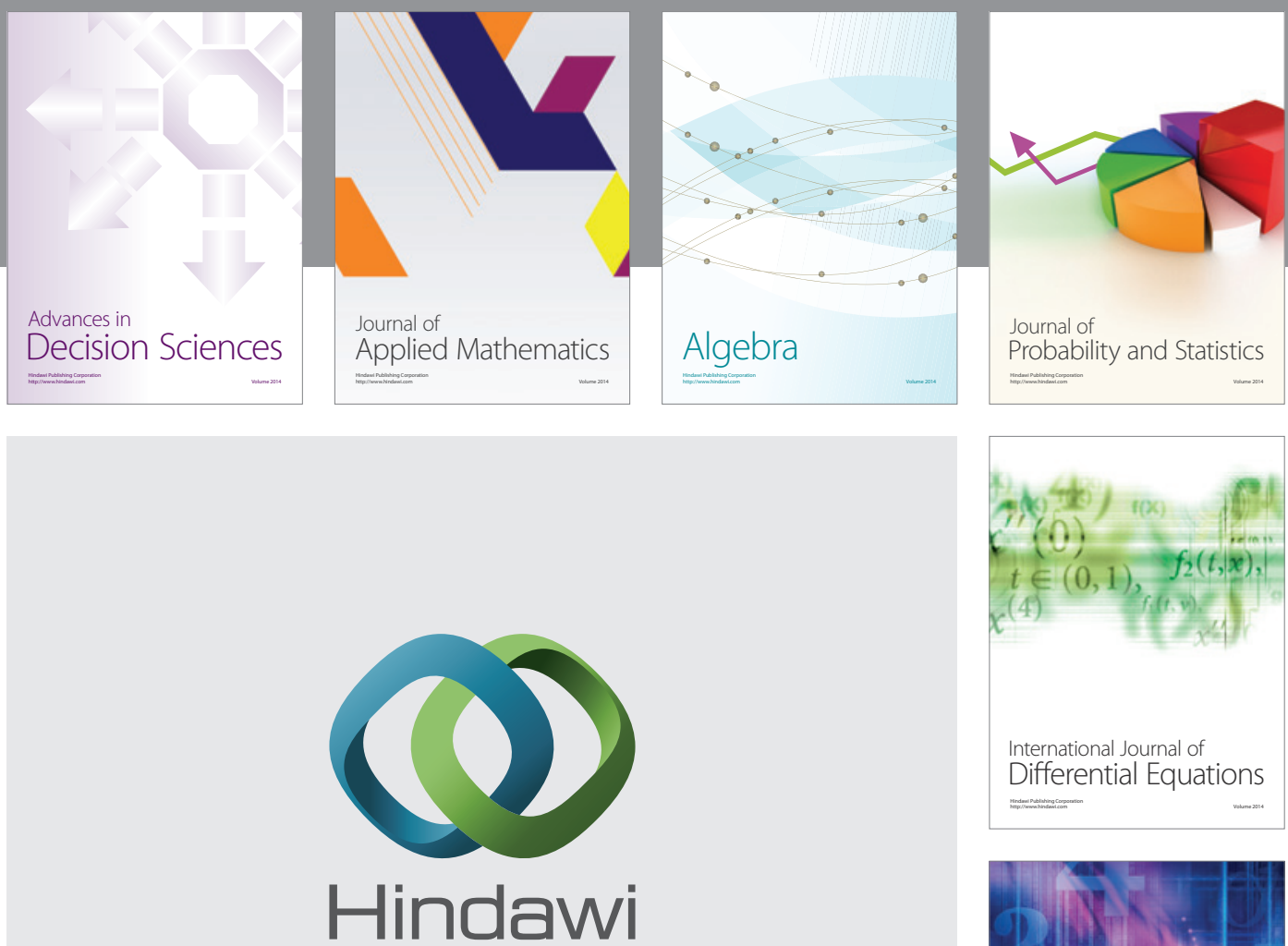

Submit your manuscripts at http://www.hindawi.com
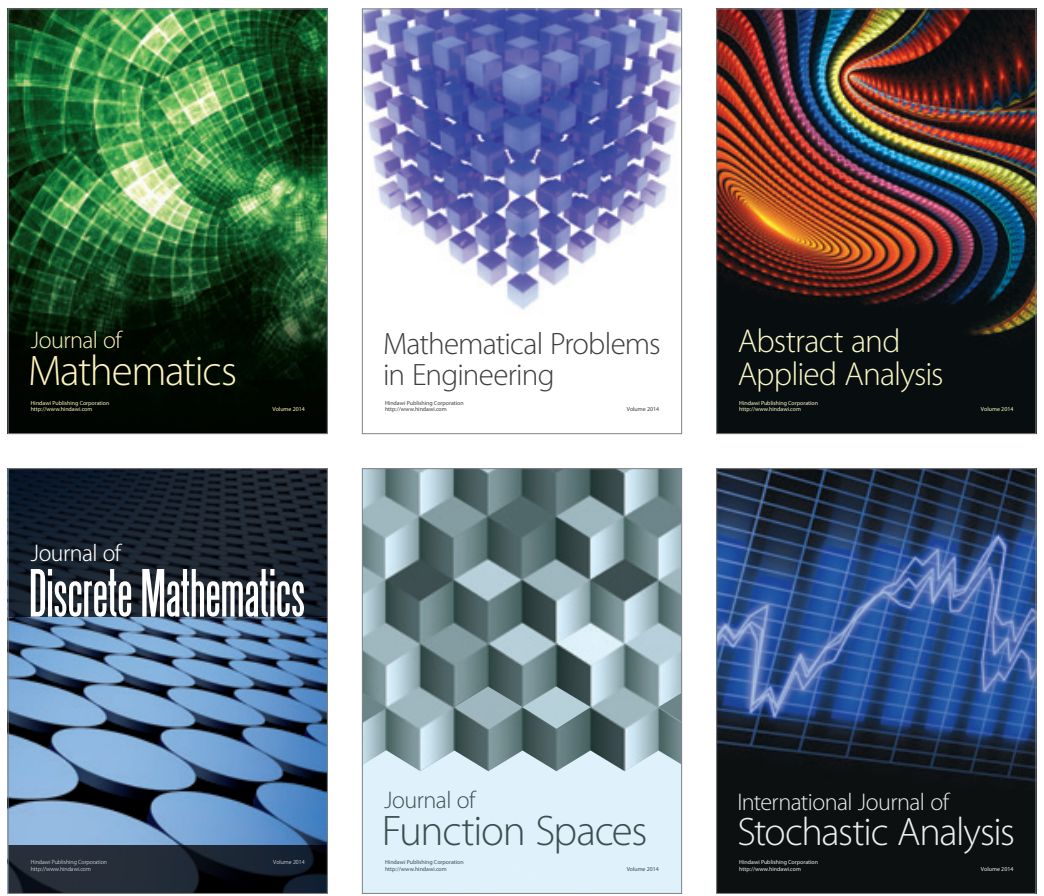

Journal of

Function Spaces

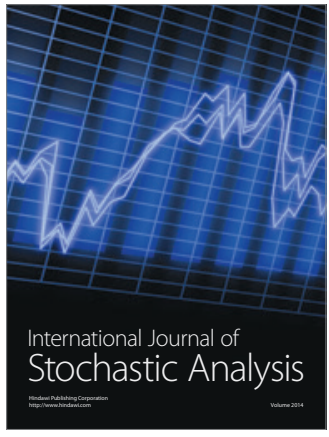

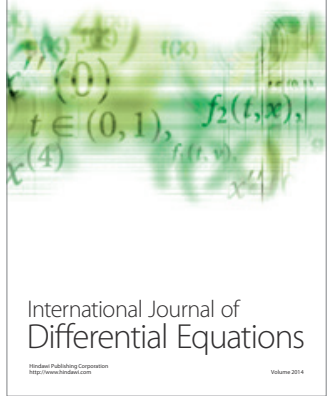
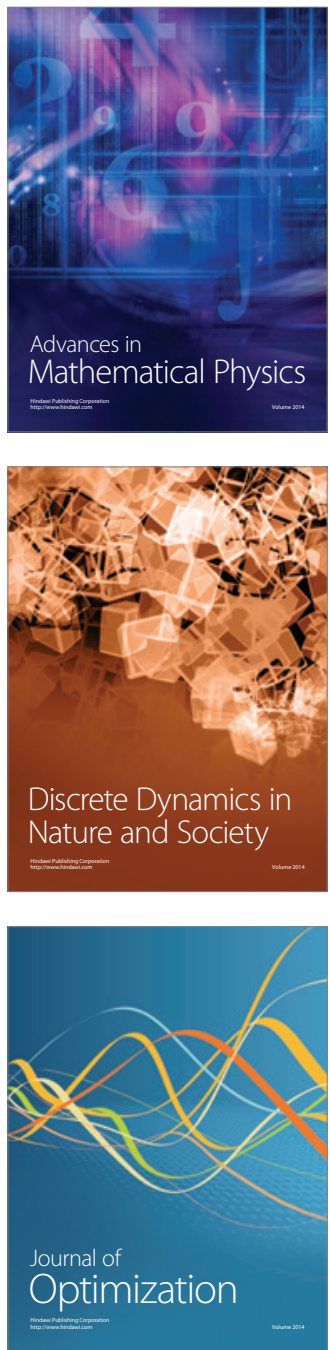